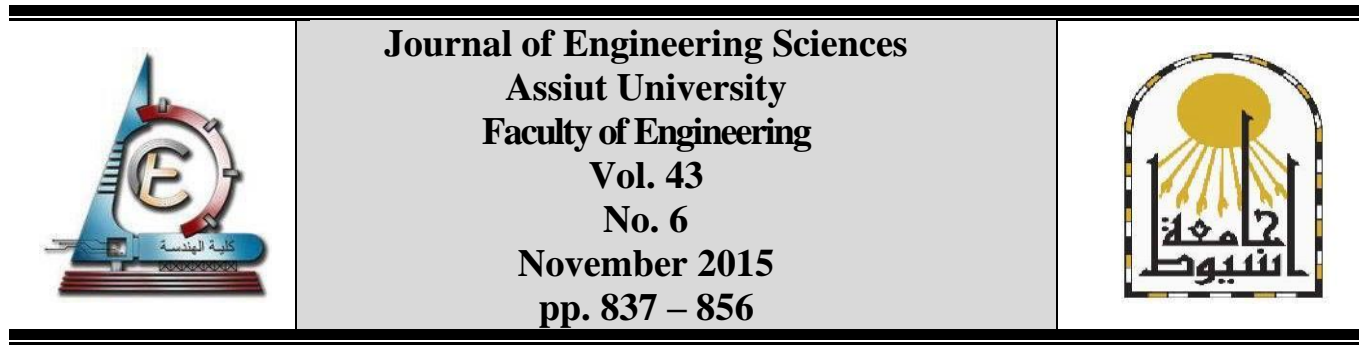

\title{
ECG SIGNAL COMPRESSION TECHNIQUE BASED ON DWT AND EXPLOITATION OF INTERBEATS AND INTRABEATS CORRELATIONS
}

\author{
Mohamed M. Abo-Zahhad ${ }^{1}$, Aziza I. Hussein ${ }^{2}$ and Abdelfatah M. Mohamed *, 3 \\ ${ }^{1}$ Engineer at Assiut University, Assiut, Egypt, (eng_mmz_eg@yahoo.com) \\ ${ }^{2}$ Depart. of Computer and Systems Eng., Faculty of Eng., Minia University,Minia, Egypt \\ ${ }^{3}$ Depart. of Electrical and Electronics Eng., Faculty of Eng., Assiut University, Assiut, Egypt
}

(Received 16 August 2015; Accepted 23 September 2015)

\begin{abstract}
A hybrid ECG compression technique based on DWT and reducing the correlation between signal samples and beats has been presented in this paper. It starts by segmenting the ECG signal into blocks; each has 1024 samples. Then, DPCM approach is adopted by removing the redundancy between successive samples. This yields to residual signal with QRS-complex like waveform without the presence of P-, T- and U-waves. Then the first QRS-complex like wave is isolated and all the succeeding ones are subtracted from the preceding ones to remove the redundancy between signal beats. The next process depends on the application. For telediagnoses, the resulting residual signal is wavelet transformed while for telemonitoring both the first QRS-complex like wave and the residual signal are wavelet transformed. In both cases the resulting wavelet coefficients are thresholded based on energy packing efficiency and coded using modified run-length algorithm. The performance of the proposed algorithm has been tested on records extracted from MIT-BIH arrhythmia database. Simulation results illustrate the excellent quality of the reconstructed signal with percentage-root-mean square difference less than $1.5 \%$ and compression ratios greater than 20 .
\end{abstract}

Keywords: ECG compression; Wavelets; Telediagnoses; Telemonitoring; Heart diseases; Arrhythmia.

\section{Introduction}

Recent trends in wireless technologies and mobile phones play an important role as an effective tool for remote health care monitoring. In the industrialized world, it is estimated that millions of people die due to various cardiac heart diseases annually. Since Electrocardiogram (ECG) is the most commonly recorded signal in the patient monitoring and examination processes, it becomes important to be able to reliably and quickly detect cardiac diseases from the ECG signal analysis. Since the maximum frequency of the ECG signal is a little bit greater than $100 \mathrm{~Hz}$, it is typically sampled at frequencies higher than $200 \mathrm{~Hz}$. Thus, a huge amount of sampled ECG data result and signal compression becomes

\footnotetext{
* Corresponding author.

Email address: afmm52@yahoo.com
} 
necessary as an important issue of research. In addition, it provides solution for the huge data handling, limited power resources and limited bandwidth in real-time transmission with low loss of significant diagnostic information.

The ECG compression system is a very essential part of telemedicine or remote analysis based health care system. However, compression distortion may cause misdiagnosis if the error between the original signal and the reconstructed one is not maintained within the tolerance range. An ECG signal contains high frequency QRS complexes and smoother $\mathrm{P}$ and $\mathrm{T}$ waves. However, there exists a redundancy between adjutant samples and between beats. ECG signal compression is achieved by elimination of such redundancies while preserving the relevant signal information.

\section{ECG signals compression techniques}

Extensive ECG signal data is not suitable for Wireless Personal Area Networks (WPAN), because the healthcare monitoring system requires real-time processes [1]. In order to tele- communicate the extensive ECG signal on WPAN, it is needed to compress the signal without loss of significant information for diagnosis. Conventional ECG compression schemes were classified into three main categories; namely the direct compression techniques, transformational compression techniques and parameters extraction techniques. Transform and parameter extraction based techniques were found to be superior to the direct compression schemes in terms of compression ratio and reconstruction quality. In the following a brief description of the main ECG compression algorithms is introduced.

\subsection{Direct ECG compression techniques}

The direct compression method detects the redundancy of an ECG signal and eliminates it in the time-domain. In other words, samples of the signal are directly used to provide the compression. The most famous methods belonging to this category are: Turning Point (TP), Amplitude Zone Time Epoch Coding (AZTEC), Coordinate Reduction Time Encoding System (CORTES), Differential Pulse Code Modulation (DPCM), and entropy encoding methods [2]-[6]. These methods give minimum distortion and are based on the elimination of redundancies by direct analysis of the original signal.

The TP data reduction algorithm has been proposed for the purpose of reducing the sampling frequency of an ECG signal to half. The simple way to do that is by considering a sample and discarding the succeeding one without reducing the elevation of the large amplitude QRS's. This yields to a compression ratio of two and retains the important features of the ECG signal. In [2], TP is achieved by replacing every three data points with the two that best represent the slope of the original three points. The second of the two saved points is used for the calculation of the next two points. A demerit of TP method is that the saved points do not represent equally spaced time intervals.

The AZTEC technique has been proposed in [3]. It converts the original ECG data into horizontal lines and slopes. The horizontal lines use a zero-order interpolator algorithm to compress the data, where amplitude and length are stored. Slopes are formed when the length of the horizontal line is less than three samples. The information stored from a slope is the length of the slope and its final amplitude. Although the AZTEC algorithm provides 
Mohamed M. Abo-Zahhad et al., ECG signal compression technique based DWT and..............

a Compression Ratio (CR) of around 10:1 but the step-like reconstruction of the ECG signal is unacceptable for accurate analysis by the cardiologist, especially in the $\mathrm{P}$ and $\mathrm{T}$ portions of the ECG signal. The CORTES algorithm is a hybrid technique that combines the high compression ratios of AZTEC algorithm and the high accuracy of the TP algorithm. The performance evaluation of the AZTEC, TP, and CORTES algorithms for ECG's sampled at $200 \mathrm{~Hz}$ is reported in [4]. Numerical results yield compression ratios of 5: 1, 2: 1, and 4.8:1 respectively, and Percentage Root-Mean-Square Difference (PRD) of 28,5 , and 7 , respectively.

In [5] the DPCM system for the ECG data compression that comes under delta coding techniques has been developed. In this system the actual signal is replaced by the firstdifference signal (amplitude between successive samples). Recently [4], a hybrid technique based on discrete-wavelet transform, DPCM, and run-length coding techniques has been presented for the compression of different parts of the ECG signal. In this technique lossless compression is adopted in clinically relevant parts and lossy compression is used in other parts of the ECG signal. In [6], Huffman variable length coding technique based on entropy encoding has been implemented for ECG signal compression. In this technique Huffman coding was applied to the frequent codeword set, while a fixed word length coding technique was applied to the infrequent set.

\subsection{Transformational ECG compression techniques}

In transformation techniques, the input signal is first preprocessed by means of linear transformation and then properly encode the transformed output for reducing the amount of data required to adequately represent the signal. For signal reconstruction, an inverse transformation is performed to get back the original signal. All transform based techniques are based on spectral and energy distribution analysis of the signal, which provide the detection of redundancy. The methods under this category are: Fast Fourier Transform (FFT), Discrete Cosine Transform (DCT), Discrete Wavelet Transform (DWT), Walsh Transformation (WT), and Karhunen-Loeve Transform (KLT) [8]-[9]. FFT is a discrete Fourier transform (DFT) algorithm which reduces the number of computations needed for a signal of length $N$ samples from $2 N^{2}$ to $2 N \log _{2} N$. In literature [10], many methods have been proposed for compressing the ECG signal. Similarly, DCT is widely exploited for ECG compression. DCT is calculated using the FFT algorithm where more weight is given to low-pass coefficients and less to high-pass ones. It gives nearly optimal performance in signals having high correlations between adjacent samples. The detailed discussion on compressing ECG signal adopting FFT and DCT is given in [11] and the references therein.

During the last decade, DWT has emerged as a robust tool for compressing ECG signals due to their time varying nature. Wavelet has localization feature along with its timefrequency resolution properties which makes it suitable for analyzing non-stationary ECG signals. Recently, several other methods have been introduced based on DWT for compressing the ECG signal [12]. A comparative study of the performance of DCT, FFT and DWT for ECG compression has been made in [13]. From this study, it has been shown that DWT is good in preserving the clinical information, while DCT and FFT give high compression ratios. 


\subsection{Parameter extraction ECG compression techniques}

The third ECG compression category is based on the extraction of particular parameters or characteristics of the signal. The extracted parameters are used for compression based on a priori knowledge of the signal features that are later used to reconstruct the signal. For example, some parameters such as measurements of the probability distribution of the original signal are extracted. Methods belonging to this category are: peak picking, linear prediction, neural networks, long term prediction, vector quantization and singular value decomposition (SVD) methods [13]-[16].

The peak picking compression technique is based on the sampling of a continuous signal at peaks (maxima and minima) and other significant points of the signal. In [13] an ECG peak-picking compression system where the signal reconstruction was achieved by using spline functions has been presented. The system finds out the points of maxima and minima, as well as those of large curvature. The performance of compression method was compared to the AZTEC method [3]. It was reported in [13] that the root-mean square error of the spline method was approximately less than that of the AZTEC method for the same CR. Among the parameters extraction ECG compression techniques, in [15] vector quantization based technique has been introduced for compressing the ECG signal while the relevant clinical information is preserved. Similarly, SVD based techniques have been presented based on the beat correlation properties of ECG signal to enhance compression performance of the algorithm [16]. In this method, low rank matrix is obtained from correlated beats of the ECG signal. Therefore, the rank truncation process computes the compressed data that is stored with fewer bits. Truncated singular value based technique has explored the different direction of low rank approximation to achieve compression for ECG data based on correlation that can be further enhanced with different encoding techniques.

\section{The proposed compression scheme}

From ECG waveform analysis, it has been found that ECG signals have quasi-periodic nature with two different correlations; namely short and full-term adjacent beat correlation and adjacent sample correlation, known as interbeat and intrabeat correlation respectively. These correlation properties play a big role in heart rate variability analysis and data compression. In the context of ECG signal compression, several algorithms have been proposed using correlation properties during the last few years, based on image processing and coding techniques [8]-[9].

The proposed ECG compression scheme is based on a hybridization of reduction of the correlation between signal samples and signal beats and DWT techniques as well as coding efficiently the decomposed signal, respectively. As these techniques have the capability to compress the signal at the cost of loss of some data in insignificant manner, they are cascaded to obtain a new ECG compression technique. Thus, the proposed hybrid technique has a good compression efficiency with good signal reconstruction quality in terms of percentage root-mean-square difference (PRD). The proposed scheme starts by segmenting the ECG signal into blocks; each of length 1024 samples. Then DPCM approach is adopted for removing the redundancy between the successive samples. The proposed scheme is clearly illustrated in Figure (1). 
Mohamed M. Abo-Zahhad et al., ECG signal compression technique based DWT and.............

\subsection{Removing redundancy between signal samples}

Most biomedical signals, including ECG signal, sampled at the Nyquist frequency or above exhibit significant correlation between successive samples. For such signals the average change in amplitude between successive samples is relatively small. Thus, an encoding scheme that exploits the redundancy in the samples is expected to result in a higher compression ratio and a lower bit rate. A relatively efficient solution to encode the differences between successive signal samples rather than the samples themselves, is the differential pulse code modulation (DPCM) technique. DPCM sequence is calculated using

$$
e(n)=x(n)-x(n-1)
$$

Where, $e(n)$ is the prediction error. A DPCM block diagram used in this study is shown in Figure (2-a). Linear Prediction (LP) coding is the direct extension of the DPCM that is aimed at predicting the value of the current sample based on the previous $\mathrm{m}$ samples, as shown in Figure (2-b).

$$
\hat{\mathrm{x}}(\mathrm{n})=\sum_{\mathrm{k}=1}^{\mathrm{m}} \mathrm{a}_{\mathrm{k}} \mathrm{x}(\mathrm{n}-\mathrm{k})
$$

Where, $\hat{x}(n)$ is the estimate of the current sample $x(n)$ at discrete time instant $\mathrm{n}$ and $\left\{a_{k}\right\}$ is the predictor weights. The samples of the estimation error sequence $(e(n)=x(n)-$ $\hat{x}(n)$ ) are less correlated with each other compared to the original signal, $x(n)$. The coefficients of the LP model are determined by minimizing the error $e(n)$ in least squares sense. In both of the above DPCM systems, $e(n)$ is normally quantized using a predefined number of bits. If the calculated difference between the current sample and its predicted value is too large to be represented by the number of bits chosen for quantization, then data loss occurs. For this reason DPCM is normally considered to be a nearly lossless coding scheme. Thus, in order to keep the DPCM operation completely lossless, the output would need more predefined bits to guarantee that there will not be data loss. Figure (3) illustrates the original ECG signal that represents the first 1024 samples of MIT-BIH record-103 and the residual signal resulting from the removal of redundancy between signal samples. From Figure (3-b) it can be observed that the residual signal contains only QRS-like waves with smaller peak-to-peak amplitude and all P-, T- and U-waves cannot be noticed.

\subsection{Removing redundancy between signal beats}

The process of removing the redundancy between ECG signal beats starts by finding the locations of the R-peaks [11]. Then the first QRS-complex like wave is isolated, saved and subtracted from the resulting residual signal. By this example the QRS-complex like wave is of 33 samples length centered around the first R-peak. Figure (4) illustrates the R-wave peaks and the isolated first QRS-complex like wave. More details of the R-wave peaks detection can be obtained from reference [7]. The next step is to remove the redundancy between the signal beats by subtracting each QRS-complex like wave from the preceding one. Figure (5) illustrates residual signal after removing the redundancy between signal samples and signal beats.

\subsection{Wavelet transformation of the residual signal}

As it can be concluded from sections 3.1 and 3.2, the ECG signal is decomposed into three parts: the first sample, the first QRS-complex like wave and the residual signal. 


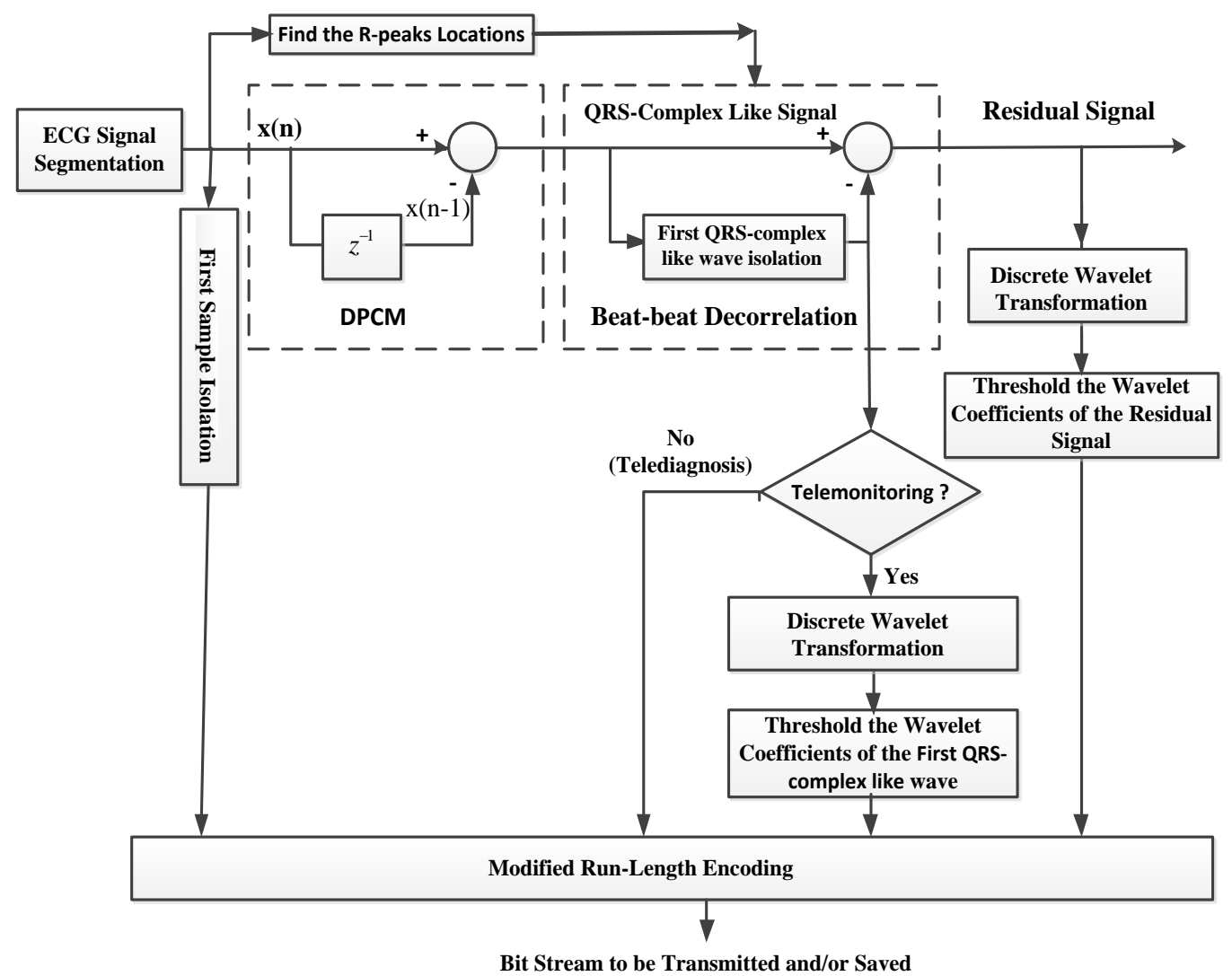

Fig. 1. Block diagram of the proposed scheme.

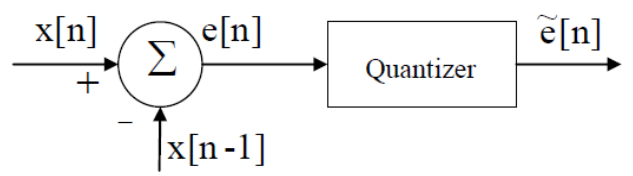

(a)

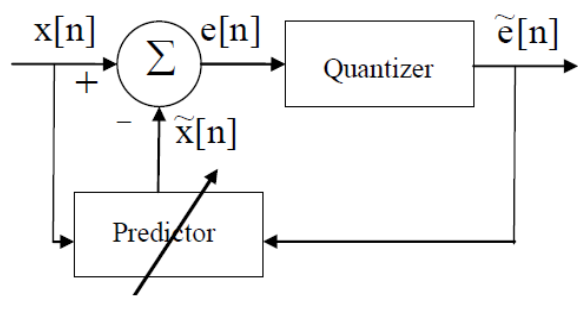

(b)

Fig. 2. DPCM - First order linear prediction model (b) mth order linear prediction model. 
(a) The original ECG signal of record-103

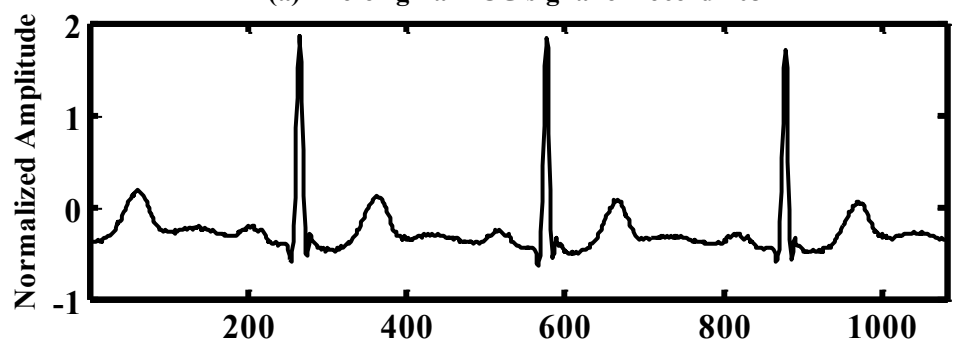

(b) The ECG signal after removing the redundency between signal samples

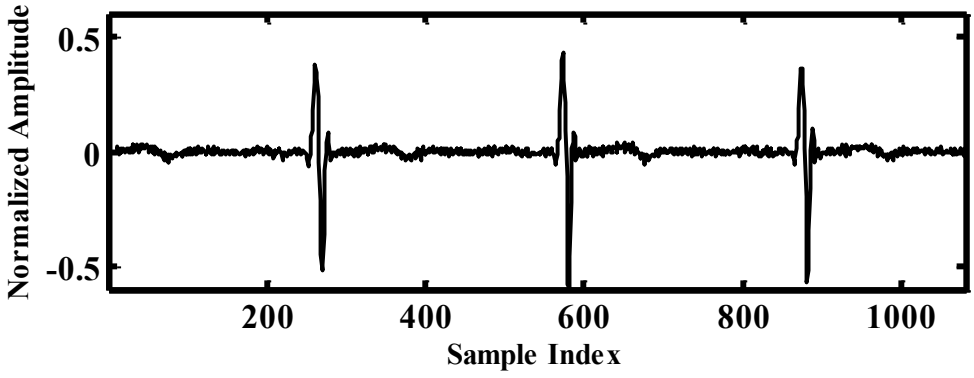

Fig. 3. Removal of redundancy between signal samples.

(a) Detecting the R-wave peaks

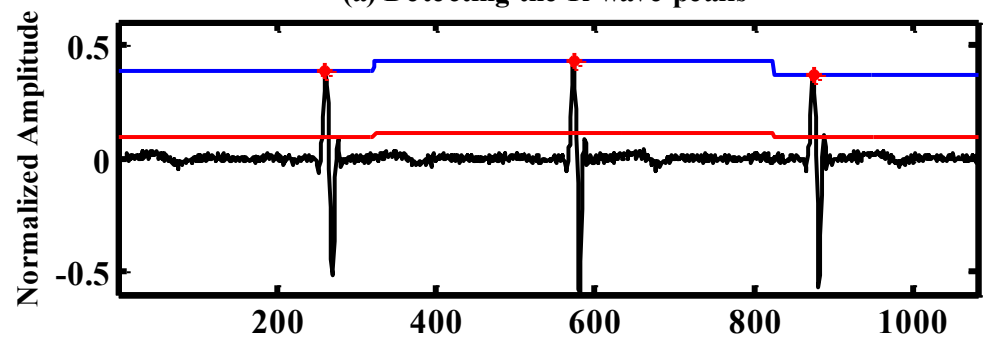

(b) Isolation of the first QRS-complex

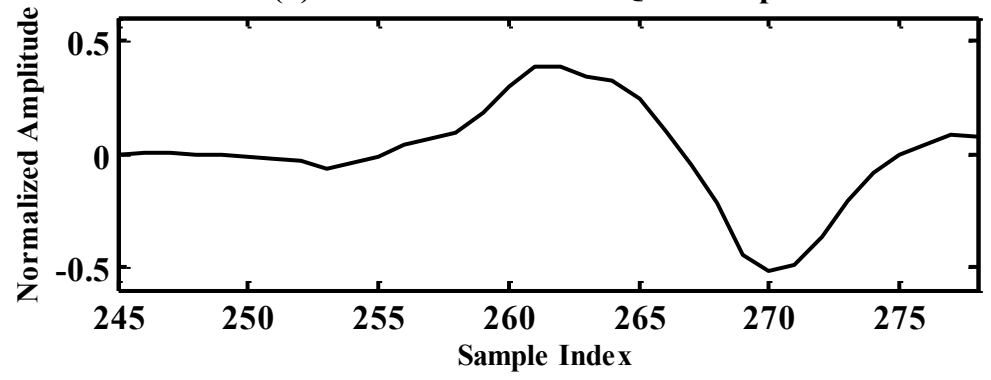

Fig. 4. Detection of the R-wave peaks and the isolation of first QRS-complex like wave. 
(a) The ECG signal after subtracting the first QRS-complex

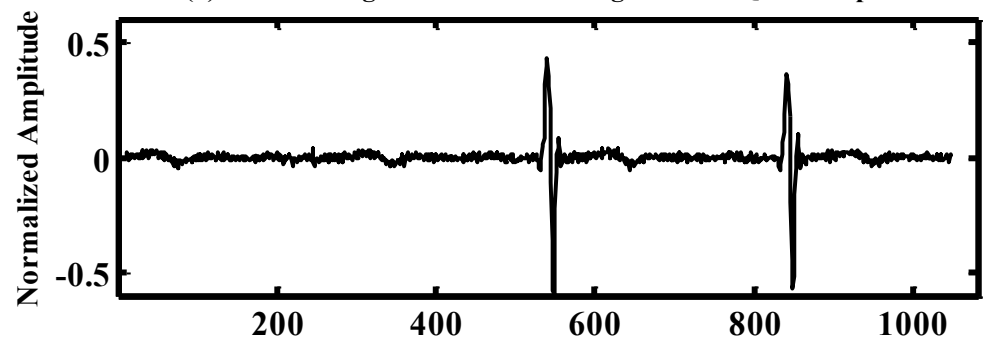

(b) Residual signal resulting from subtracting precceding QRS-complexes

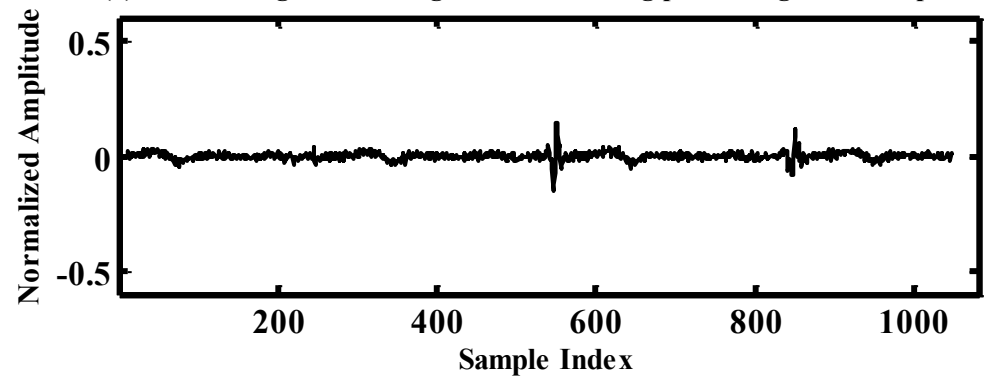

Fig. 5. Removing the redundancy between signal samples and signal beats.

Table (1) includes the main features of the three components as well as that of the original ECG signal shown in Figure (3-a). It is quite clear from the table that $87.47 \%$ of the total signal energy is concentrated in 34 signal samples and the remaining $12.53 \%$ of the energy is concentrated in 1048 residual samples. In addition the amplitudes of the residual samples are much less than that of the first sample and the first QRS-complex like wave. The decision of how the decomposed signal parts are manipulated depends on the applications. If the signal is exploited for telediagnoses, the first sample, and the first QRScomplex like wave are kept without thresholding and wavelet transformation. However, if the signal is utilized for telemonitoring, both the first QRS-complex like wave and the residual signal are wavelet transformed and thresholding is allowed. Many wavelet families and families members have been developed so far for signal and image compression [7]. However, Daubechies, Biorthogonal, Symlets and Coiflets families have been extensively adopted for this purpose. Among the large wavelet families members db6, bior3.7 and coif5 filters have been proved efficient for ECG signal compression applications. Thresholding has been carried out by discarding the WT-coefficients, which are less than a given threshold level determined such that $97 \%$ of the total energy is kept. Compression is then achieved by efficiently coding the thresholded WT-coefficients.

\subsection{Modified run-length encoding}

The main aim of the modified run-length encoding introduced here is to represent the theresholded wavelet coefficients with a small number of bits. It is based on grouping these coefficients into significant and insignificant. 
Mohamed M. Abo-Zahhad et al., ECG signal compression technique based DWT and..............

\section{Table 1.}

The main features of the decomposed and the original ECG signals.

\begin{tabular}{|c|c|c|c|c|}
\hline \multirow{2}{*}{ Feature } & \multicolumn{3}{|c|}{ Decomposed ECG signal } & \multirow{2}{*}{$\begin{array}{c}\text { Original } \\
\text { ECG signal }\end{array}$} \\
\cline { 2 - 4 } & $\begin{array}{c}\text { First } \\
\text { sample }\end{array}$ & $\begin{array}{c}\text { First QRS-complex } \\
\text { like wave }\end{array}$ & Residual signal & \\
\hline Length (number of samples) & 1 & 33 & 1048 & 1080 \\
\hline Energy & 0.1406 & 1.6720 & 0.2593 & 168.88 \\
\hline$\%$ (Energy / Total Energy) & $6.78 \%$ & $80.70 \%$ & $12.52 \%$ & $100 \%$ \\
\hline Maximum value & -0.3750 & 0.3800 & 0.1251 & 1.8500 \\
\hline Minimum value & -0.3750 & -0.5200 & -0.1457 & -0.6350 \\
\hline
\end{tabular}

The nonzero valued $\mathrm{N}_{S}$ coefficients are considered significant and the remaining $\mathrm{N}_{I}$ zero valued coefficients are considered insignificant; where $\mathrm{N}_{I}=\mathrm{N}-\mathrm{N}_{S}$ and $\mathrm{N}$ is the total number of theresholded coefficients. Most of the insignificant coefficients are located at the end of the coefficients vector. The algorithm is based on representing each significant coefficient by $b_{S}+1$ bits and the insignificant coefficients are manipulated in a different manner. First, the repeated groups of zeros are counted and the resulting count is represented by $b_{S}+1$ bits. Then the train of coefficients representing the ECG signal is transformed to another train of numbers. Some of these numbers represent the significant coefficients and the rest represent the repeated group of zeros $\left(K_{1}, K_{2}, \ldots, K_{M}\right)$. Here, $M$ denotes the number of these groups. The problem here is how to differentiate between the coefficients and the numbers representing the group of zeros. For example, the number 18 may appear twice in the new train of numbers, where the first 18 may be a significant coefficient and the second one may indicate 18 repeated zeros. To overcome this problem, a prefix bit in the representation of each number is added as a control bit. In case of significant coefficient this bit is set to one and in case of repeated zeros it is reset to zero. Table (2) includes the encoding of significant and insignificant residual coefficients; where each coefficient is represented by $b_{S}+1$ bits.

Table 2.

Encoding of significant and insignificant coefficients.

\begin{tabular}{|l|c|c|}
\hline \multirow{2}{*}{ Type of the coefficients } & \multicolumn{2}{c|}{ Representation } \\
\cline { 3 - 4 } & Prefix & Number of bits \\
\hline - Significant residual coefficient & 1 & $\mathrm{~b}_{\mathrm{s}}-\mathrm{bits}$ \\
\hline - Insignificant residual coefficient (group of repeated zeros) & 0 & $\mathrm{~b}_{\mathrm{s}}-\mathrm{bits}$ \\
\hline
\end{tabular}

\section{Performance measures}

The overall performance of the ECG compression algorithms is measured using three important parameters; namely the Compression Ratio (CR) defined as the ratio between the number of bits needed to represent the original and the number of bits needed to represent the compressed signal; the reconstruction error and the computational complexity. The reconstruction error is defined as the difference between the original signal and the reconstructed one. It measures the ability of the reconstructed signal to preserve the relevant diagnostic information; any slight loss or change of information can 
lead to wrong diagnosis. In the case of ECG compression, data that does not contain diagnostic information can be removed. The computational complexity component relates to practical implementation considerations and is desired to be as low as possible. In [8] most ECG compression algorithms evaluate the errors using PRD that is defined by the square root of the ratio between reconstructed noise energy and the original signal energy.

$$
\% P R D=\sqrt{\frac{\sum_{n=1}^{N}(x(n)-\widehat{x}(n))^{2}}{\sum_{n=1}^{N} x^{2}(n)}} * 100
$$

Where, $x(n)$ and $\widehat{x}(n)$ are respectively the original and reconstructed signals and $\mathrm{N}$ is the length of the window over which the PRD is calculated [14]. This error estimate is the one most commonly used in all scientific literature concerned with ECG compression techniques. The main drawbacks are the inability to cope with baseline fluctuations and the inability to discriminate between the diagnostic portions of an ECG curve. However, its simplicity and relative accuracy make it a popular error estimate among researchers [8]-[9]. In [25], it has been shown that the globally considered standard of ECG signal reconstruction quality in terms of PRD is defined as PRD < 2\%: very good reconstruction, PRD <2-5\%: good reconstruction, PRD < 5-9\%: acceptable quality of reconstruction and PRD > 9\%: unacceptable quality. Here, it has been considered that very good reconstruction can be adopted for telediagnoises applications and the other cases for telemonitoring applications. The computation of both CR and PRD requires the reconstruction of the compressed transmitted signal. The proposed scheme used on the receiver side is clearly illustrated in Figure (6).

The reconstructed residual signal before and after adding the first QRS-complex like wave is illustrated in Figure (7). Figure (8) illustrated the reconstructed residual signal after adding all QRS-complex like waves and the first sample. Figures (9-a) and (9-b) show the comparison between the original and reconstructed ECG signals and the difference between them respectively.

\section{Results and discussion}

In the field of ECG signal compression, several techniques have been reported in the literature [18]-[20]. In order to promote the proposed algorithm, it is necessary to compare it with other satisfactory published results. For this purpose, the proposed method is tested on two signals extracted from records 100 and 117 of the MIT-BIH database [24]; for which the sampling rate is $360 \mathrm{~Hz}$ and the resolution is 11-bit. The comparison with the existing methods has been made in term of higher compression performance and lower signal distortion. It is evident that the proposed technique has shown its efficiency over other existing techniques. Tables (3) and (4) include the comparative performance study of the proposed method and the earlier published algorithms [16]-[23]. The simulated experimental results of the proposed algorithm are obtained using $\mathrm{db} 5$ and bior3.7 wavelet filters for record MIT-BIH 100 record MIT-BIH 117 respectively. 
Received Bit Stream

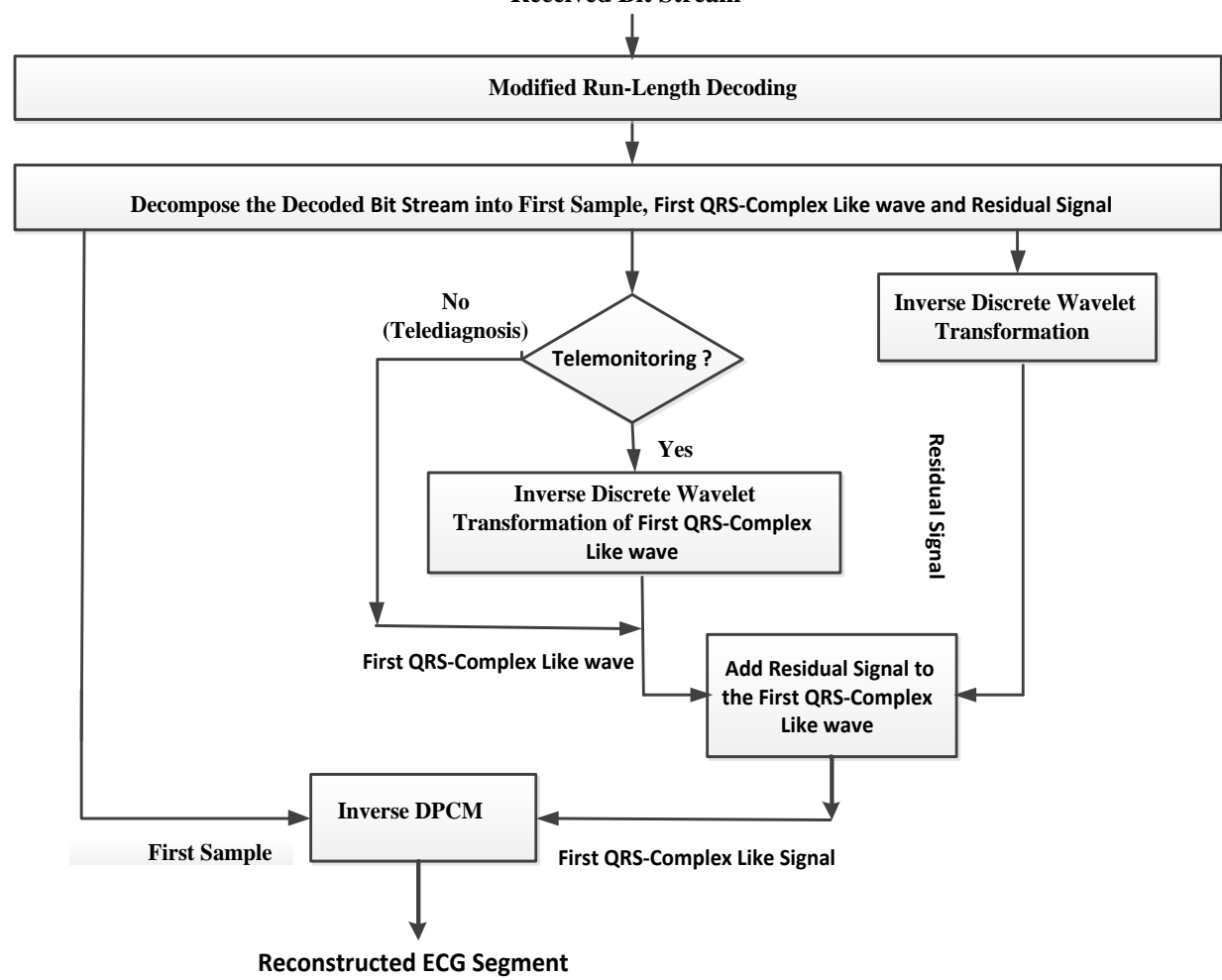

Fig 6. Block diagram of the proposed scheme - receiver part.

(a) The reconstructed residual ECG signal

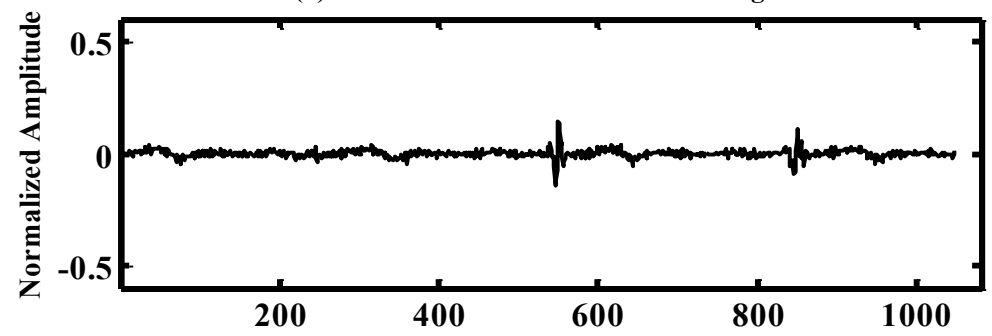

(b) The reconstructed residual signal after adding the first QRS-complex

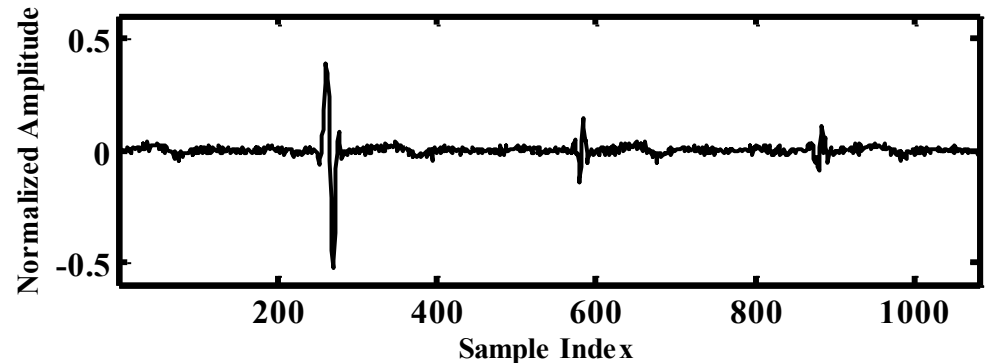

Fig. 7. Reconstructed residual signal with and without the first QRS-complex like wave. 
(a) The reconstructed signal after adding the precceding QRS-complexes

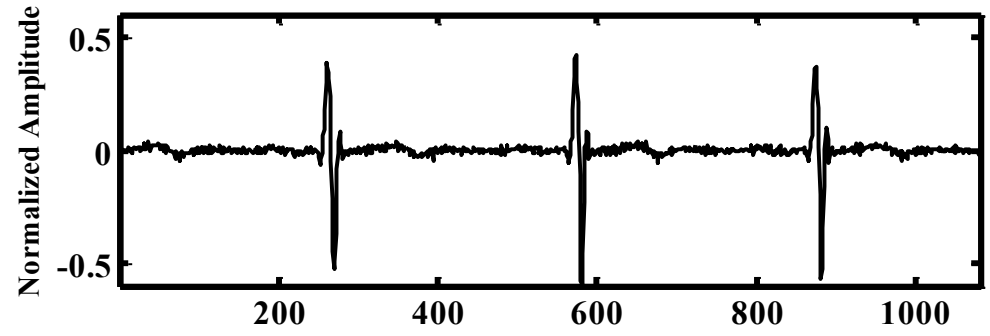

(b) The reconstructed signal after adding precceding QRS-complexes and 1st sample

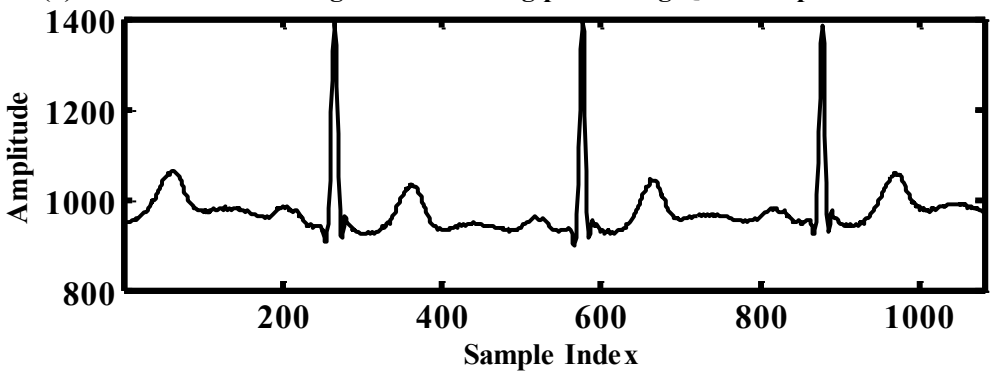

Fig. 8. Reconstructed residual signal after adding all QRS-complex like waves and 1st sample.

(a) Comparison between original (solid) and reconstructed (dotted) ECG signals

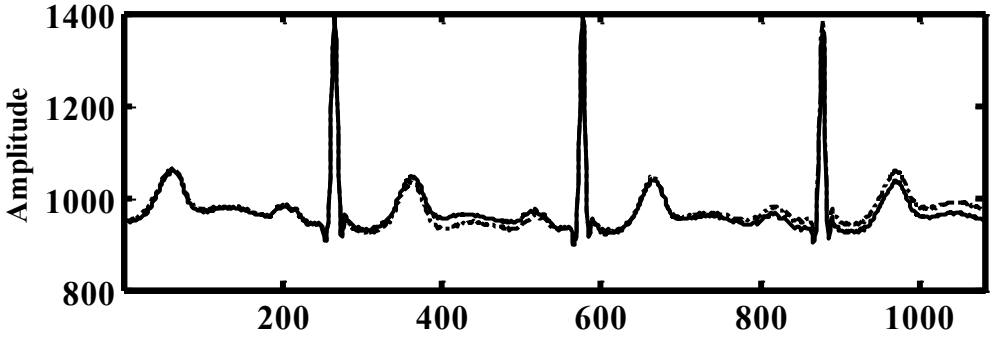

(b) The Difference between the original and reconstructed ECG signals

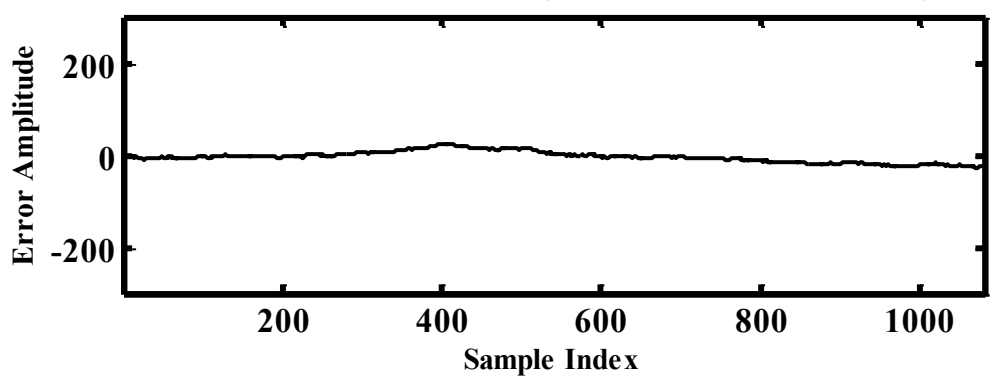

Fig. 9. (a) Comparison between the original and reconstructed ECG signals; (b) The difference between the original and reconstructed ECG signals.

Here, it should be noted that for the comparison between different methods to be complete, the computational complexity should be done. However, this has not been done here since neither the computational time nor the software code of the earlier published 
Mohamed M. Abo-Zahhad et al., ECG signal compression technique based DWT and..............

algorithms is available in these publications [16]-[23]. Figures (10)-(13) illustrate the original, reconstructed and error signals for records 100 and 117 respectively. From Figures (10) and (12), it can be seen that the original and reconstructed ECG signals are approximately matched. Thus, they can be used for telediagnosis applications. However, Figures (11) and (13) show that differences between the original and reconstructed ECG signals are noticeable; thus they can be used for telemonitoring applications.

Table 3.

Comparison between the proposed method and earlier published algorithms for record MIT-BIH 100.

\begin{tabular}{|c|c|c|c|c|}
\hline Method & CR & PRD (\%) & $\begin{array}{c}\text { Reconstructed signal } \\
\text { quality }\end{array}$ & Possible Application \\
\hline $\begin{array}{c}\text { Tai et al. (2005) } \\
{[17]}\end{array}$ & 5 & 1.05 & very good & Telediagnoises \\
\cline { 2 - 5 } & 10 & 1.48 & very good & Telediagnoises \\
\hline $\begin{array}{c}\text { Huang et al. } \\
\text { (2009) 18] }\end{array}$ & 8 & 1.65 & very good & Telediagnoises \\
\hline $\begin{array}{c}\text { Ciocoiu (2009) } \\
{[19]}\end{array}$ & 20.9 & 3.14 & Good & Telemonitoring \\
\hline Proposed Method & 10.48 & 1.71 & very good & Telediagnoises \\
\cline { 2 - 5 } & 22.98 & 2.65 & very good & Telediagnoises \\
\hline
\end{tabular}

\section{Table 4.}

Comparison between the proposed method and earlier published algorithms for record MIT-BIH 117.

\begin{tabular}{|c|c|c|c|c|}
\hline Method & $\mathrm{CR}$ & $\operatorname{PRD}(\%)$ & $\begin{array}{c}\text { Reconstructed signal } \\
\text { quality }\end{array}$ & Possible Application \\
\hline Wei et al. (2001) [16] & 10 & 1.188 & very good & Telediagnoises \\
\hline \multirow{2}{*}{ Tai et al. (2005) [17] } & 5 & 0.42 & very good & Telediagnoises \\
\hline & 10 & 0.67 & very good & Telediagnoises \\
\hline $\begin{array}{l}\text { Abo-Zahhad et.al } \\
\text { (2012) [20] }\end{array}$ & 19.20 & 1.60 & very good & Telediagnoises \\
\hline $\begin{array}{l}\text { Abo-Zahhad et.al } \\
\text { (2013) [21] }\end{array}$ & 23.10 & 1.60 & very good & Telediagnoises \\
\hline $\begin{array}{c}\text { Kumar et. al (2013) } \\
{[22]}\end{array}$ & 5.95 & 2.50 & good & Telemonitoring \\
\hline $\begin{array}{c}\text { Hung et. al (2014) } \\
{[23]}\end{array}$ & 22.71 & 2.88 & good & Telemonitoring \\
\hline \multirow{2}{*}{ Proposed Method } & 23.86 & 2.76 & good & Telemonitoring \\
\hline & 7.02 & 1.48 & very good & Telediagnoises \\
\hline
\end{tabular}

\section{Conclusions}

Compression can significantly reduce the cost of medical information transmission through telecommunications channels. An ECG signal has quasi-periodic nature which represents the considerable correlation between the adjacent heartbeats as well as short-term correlation between the adjacent samples. In this paper, a hybrid compression scheme based on reducing the correlation between signal samples and signal beats as well as DPCM and DWT techniques. 
The proposed algorithm is tested adopting records extracted from MIT-BIH Arrhythmia Database considering the compression ratio and the PRD distortion metrics. Simulation results have clearly shown the effectiveness of the proposed algorithm in getting high compression ratios with acceptable reconstruction signal quality compared to recently published results [16][23]. It is flexible in dealing with different types of applications; namely telediagnoses and telemonitoring of cardiac patients. Moreover, it can play a big role to save memory space of health data centers and save bandwidth in telemedicine based health care systems.

\section{REFERENCES}

[1] H. Kim, R. F. Yazicioglu, P. Merken, C. V. Hoof, and H. Yoo, "ECG signal compression and classification algorithm with quad level vector for ECG Holter system," IEEE Trans. Inf. Technol. Biomed., vol. 14, pp. 93-100, 2010.

[2] W. C. Mueller, "Arrhythmia detection program for an ambulatory ECG monitor," Biomed. Sci. Instrum., vol. 14, pp. 81-85, 1978.

[3] J. R. Cox, F. M. Nolle, H. A. Fozzard, and G. C. Oliver Jr., "AZTEC, a preprocessing program for real time ECG rhythm analysis,” IEEE Trans. Biomed. Eng., vol. 15, pp. 128-129, 1968.

[4] J. Abenstein and W. Tompkins, "A new data-reduction algorithm for realtime ECG analysis," IEEE Trans. Biomed. Eng., vol. BME-29, pp. 43-48, 1982.

[5] U. E. Ruttimann and H. V. Pipberger, "Compression of the ECG by prediction or interpolation and entropy encoding," IEEE Trans. Biomed. Eng., vol. BEM-26, pp. 613-623, 1979.

[6] S A Chouakri, O Djaafri1 and A Taleb-Ahmed, "Wavelet transform and Huffman coding based electrocardiogram compression algorithm: Application to telecardiology," IOP Publishing, Journal of Physics: Conference Series, vol. 454, pp. 1-16, 2013.

[7] M. M. Abo-Zahhad, Tarik K. Abdel-Hamid, Abdelfatah M. Mohamed. "Compression of ECG signals Based on DWT and Exploiting the Correlation between ECG Signal Samples" Int. J. Communications, Network and System Sciences, vol. 7, no. 2, pp. 101-118, January 2014.

[8] M. Abo-Zahhad, "ECG Signal Compression Using Discrete Wavelet Transform," Chapter in "Discrete Wavelet Transforms - Theory and Applications," Edited by J. T. Olkkonen, InTech, 2011.

[9] S. M. S., Jalaleddine, Ch. G., Hutchens, R. D., Stratton and W. A. Coberly, "ECG Data Compression techniques - A Unified Approach," IEEE Trans. on Biomedical Engineering, vol. 37, no. 4, pp. 329-343, 1999.

[10] A. A. Iglesias and J. G. Moros, "Automatic real time ECG coding methodology guaranteeing signal interpretation quality,” IEEE Trans. Biomed. Eng., vol. 55, pp. 2519-2527, 2008.

[11] V. Aggarwal and M. S. Patterh, "Quality Controlled ECG Compression using Discrete Cosine Transform (DCT) and Laplacian Pyramid (LP)," Multimedia, Signal Processing and Communication Technology, pp. 12-15, 2009.

[12] Sabah M. Ahmed and M. Abo-Zahhad, "A new hybrid algorithm for ECG signal compression based on the wavelet transformation of the linearly predicted error" Medical Engineering and Physics, vol. 23, pp. 117-126, 2001.

[13] K. Ranjeet, A. Kumar, and R.K. Pandey "ECG Signal Compression Using Different Techniques" ICAC3 2011, pp. 231-241, 2011.

[14] A. Al-Shrouf, M. Abo-Zahhad, S.M. Ahmed, "A novel compression algorithm for electrocardiogram signals based on the linear prediction of the wavelet coefficients," Digital Signal Processing, vol. 13, pp. 604-622, 2003.

[15] C. P. Mammen and B. Ramamurthi, "Vector quantization for compression of multichannel ECG" IEEE Transactions on Biomedical Engineering. vol. 37, pp. 821-825, 1990.

[16] J. J. Wei, Ch. J. Chang, N. K. Chou and G. J. Jan, "ECG data compression using Truncated Singular value decomposition," IEEE Trans. on Info. Tech. in Biomed., vol. 5, pp. 290-299, 2001. 
[17] S. Ch. Tai, Sun, and W. Ch. Yan, "A 2-D ECG compression method based on wavelet transform and modified SPIHT," IEEE Trans. on Biomedical Eng. 52 (6), 999-1008, 2005.

[18] Huang, B., Wang, Y. (2009). 2-D compression of ECG signals using ROI mask and Conditional Entropy Coding. IEEE Trans. on biomedical engineering, 56 (4), 1261-1263

[19] I. B. Ciocoiu, "ECG Signal compression using 2D wavelet Foveation," Int. J. of Advanced Science and Technology, pp. 15-26, 2009.

[20] M. Abo-Zahhad, S. M. Ahmed and A. Zakaria, "An Efficient Technique for Compressing ECG Signals Using QRS Detection, Estimation, and 2D DWT Coefficients Thresholding," Modeling and Simulation in Engineering, 2012, Doi:10.1155/2012/742786.

[21] M. Abo-Zahhad, A. F. Al-Ajlouni, S. M. Ahmed, and R. J. Schilling, "A new algorithm for the compression of ECG signals based on mother wavelet parameterization and bestthreshold levels selection," Digital Signal Processing, vol. 23, pp. 1002-1011, 2013.

[22] R. Kumar, A. Kumar and R. K. Pandey, "Beta wavelet based ECG signal compression using lossless encoding with modified thresholding," Computer and Electrical Engineering, vol. 39, no. 1, pp. 130-140, 2013.

[23] K-Ch., Hung, T-Ch., Wu, H-W., Lee and T-K. Liu, "EP-based wavelet coefficient quantization for linear distortion ECG data compression," Medical Engineering \& Physics, vol. 36, pp. 809-821, 2014.

[24] MIT-BIH Arrhythmia database (www.physionet.org/ physiobank/database/mitdb ).

[25] Y. Zigel, A., Cohen and A., Katz, "The weighted diagnostic distortion measure for ECG signal compression," IEEE Trans. Biomed. Eng., vol. 47, no. 11, pp. 1422-1430, 2000. 


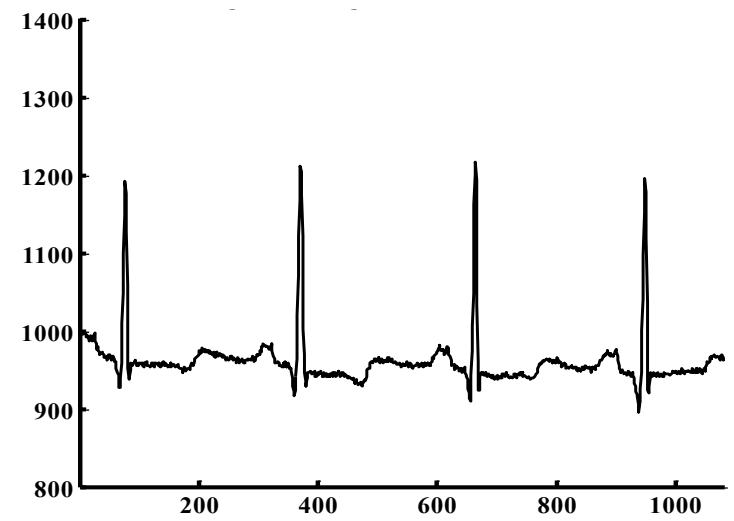

(a) Original ECG signal extracted from MIT-BIH record 100.

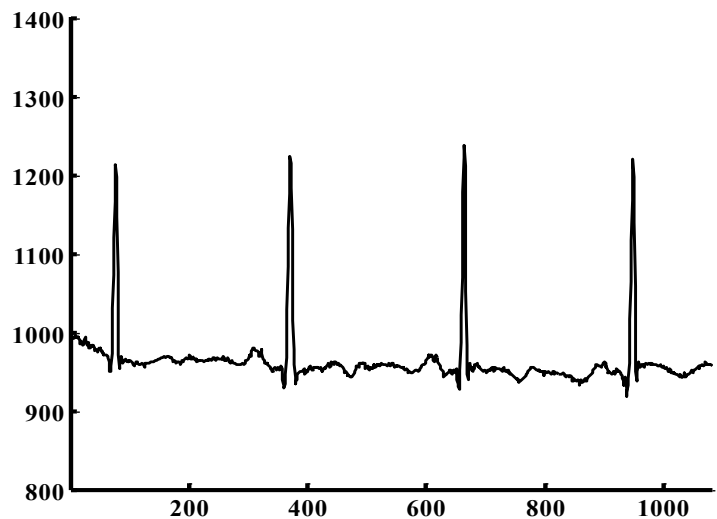

(b) Reconstructed ECG signal extracted from MIT-BIH record 100 (CR=10.48 and PRD\%=1.71\%).

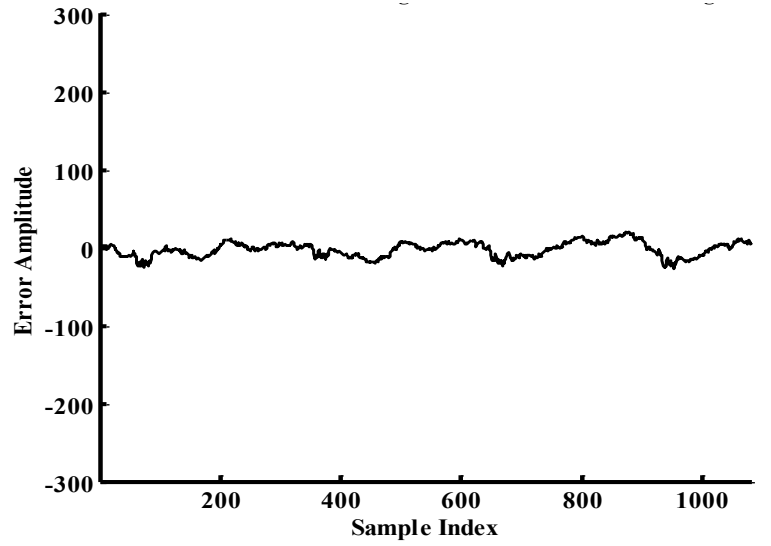

(c) The differences the original and reconstructed ECG signals.

Fig. 10. Compressing ECG signal extracted from MIT-BIH record 100 for telediagnoses applications. 


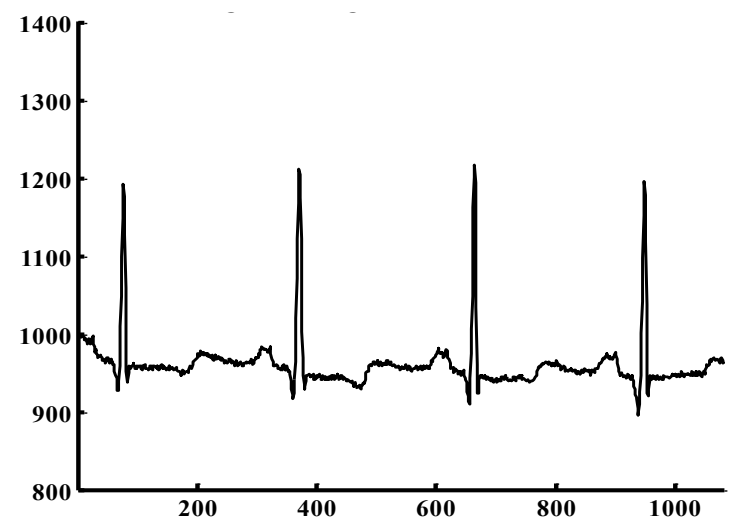

(a) Original ECG signal extracted from MIT-BIH record 100.

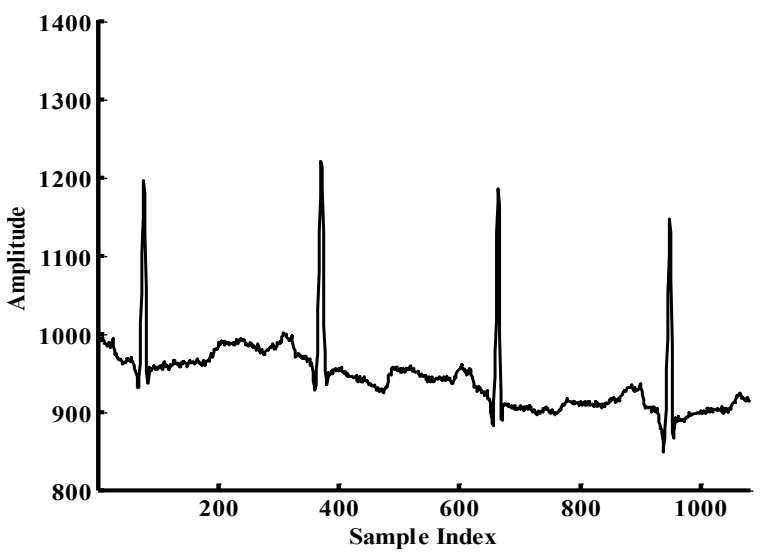

(b) Reconstructed ECG signal extracted from MIT-BIH record 100 (CR=22.98 and PRD\%=2.65\%).

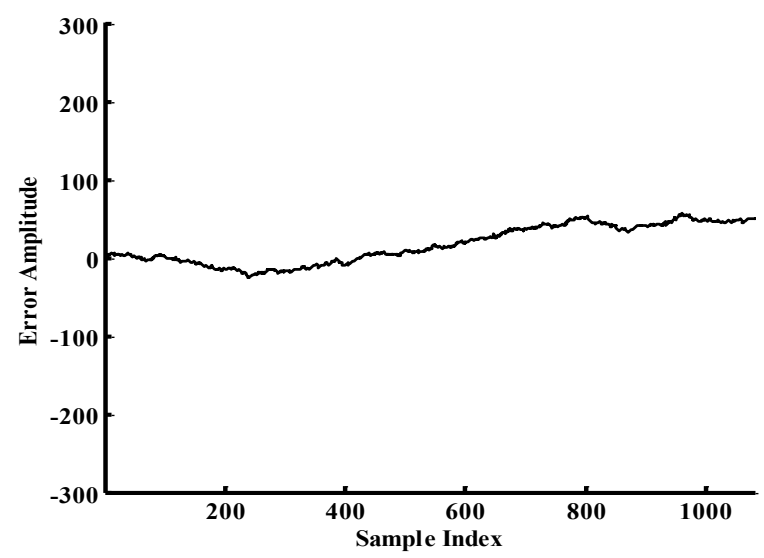

(c) The differences the original and reconstructed ECG signals.

Fig. 11. Compressing ECG signal extracted from MIT-BIH record 100 for telemonitoring applications. 


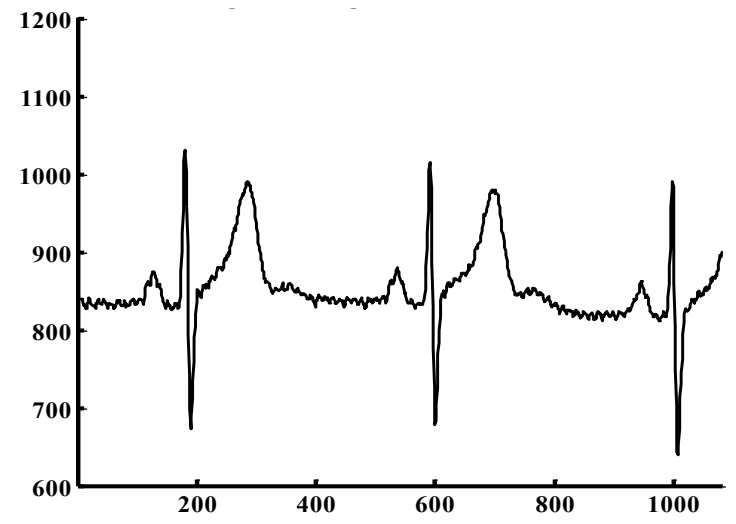

(a) Original ECG signal extracted from MIT-BIH record 117.

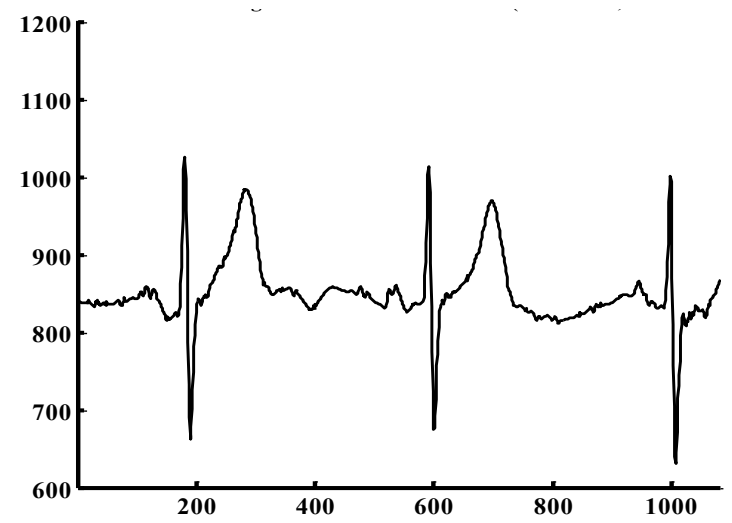

(b) Reconstructed ECG signal extracted from MIT-BIH record 117 (CR=20.02 and PRD\%=1.48 \%).

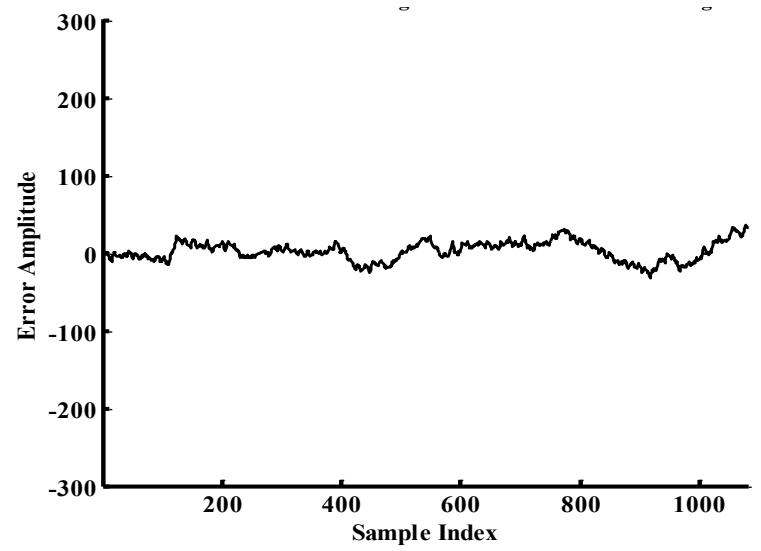

(c) The differences the original and reconstructed ECG signals.

Fig. 12. Compressing ECG signal extracted from MIT-BIH record 117 for telediagnoses applications. 


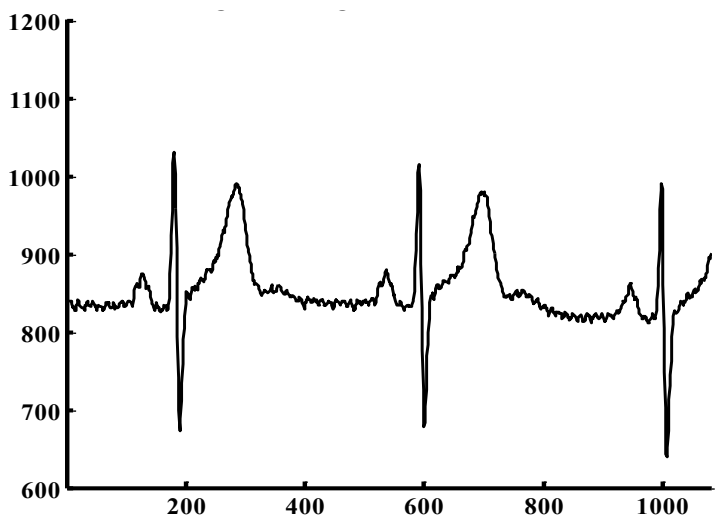

(a) Original ECG signal extracted from MIT-BIH record 117.

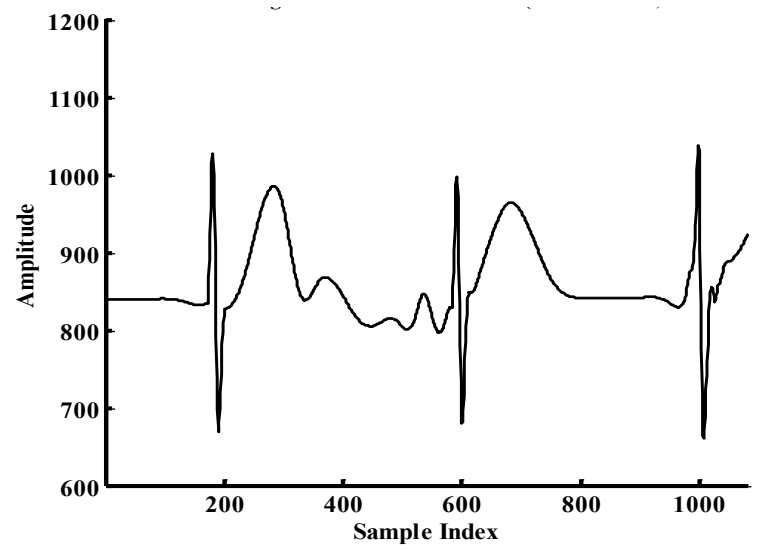

(b) Reconstructed ECG signal extracted from MIT-BIH record 117 (CR=23.86 and PRD\%=2.76 \%).

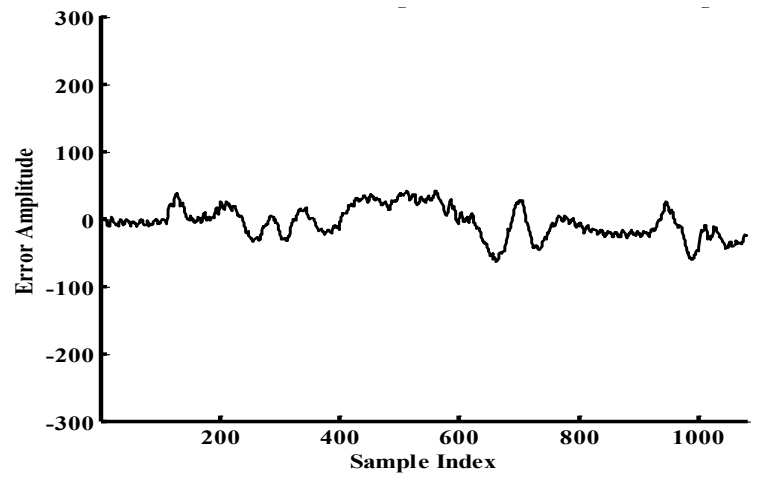

(c) The differences the original and reconstructed ECG signals.

Fig. 13. Compressing ECG signal extracted from MIT-BIH record 117 for telemonitoring applications. 


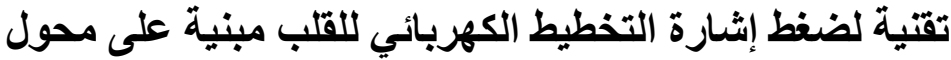 \\ المويجة المتقطع واستغلال الارتباطات بين عينات الآشارة بين التبط التبضات المتجاورة \\ الملخص العربى:}

يقدم البحث تقنية جديدة لضغط إثنار ات التخطيط الكهربائي للقلب مبنية على تحويل المويجة المتقطع واستغلال

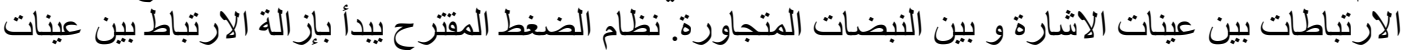
الإشارة حيث ينتج عن ذللك إثـارة شبيهه بالموجة المعقدة QRS و الإنات تختفي باقي موجات الثارة التخطيط

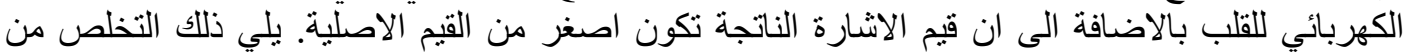

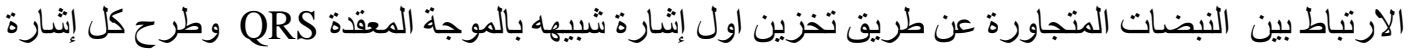
شبيهه بالموجة المعقدة QRS من التالية لها. و علاوة على ذللك، يتم تحويل الإشارة المتبقية الناتجة باستخدام

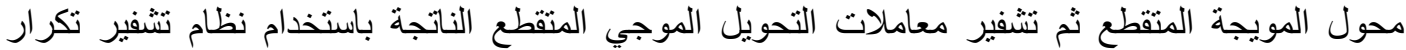

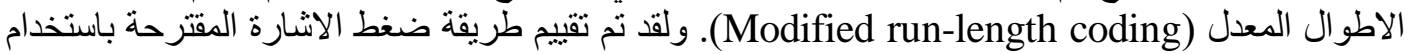

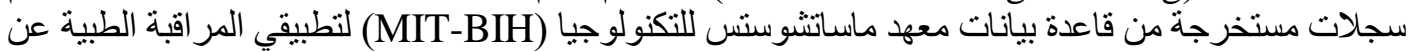

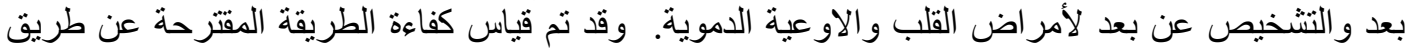
مقارنتها مع الطرق المثيلة السابق نشرها. واظهرت نتائج المفارنة تميز الطريقة المقترحة من حيث نسبة

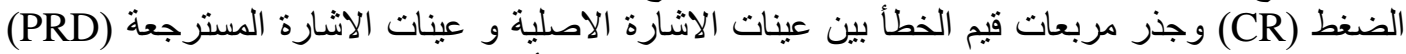

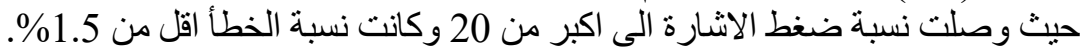

